\title{
UKIRT Widefield Infrared Survey for $\mathbf{H}_{2}$
}

\section{Dirk Froebrich, Georgios Ioannidis and UWISH2 Survey Team}

\author{
University of Kent, INGRAM Building, Canterbury CT2 7NH, UK \\ email: df@star.kent.ac.uk
}

\begin{abstract}
We perform an unbiased search for outflows from YSOs along the Galactic Plane (GP). Our data has been taken as part of the UWISH2 survey (Froebrich et al. 2011). It uses as tracer the 1-0S(1) emission line of $\mathrm{H}_{2}$, and here we focus on a continuous 33 square degree sized region in Serpens and Aquila. We identify 130 outflows from which $94 \%$ are new discoveries. Thus, we increased the number of known MHOs by a factor of 15 in this area (Ioannidis \& Froebrich 2012). The flux completeness limit for the flows is $3 \cdot 10^{-18} \mathrm{Wm}^{-2}$. Typically, the known flows occupy the bright end of the flux distribution. Our survey thus increases the known integrated 1-0S $(1) \mathrm{H}_{2}$ flux from outflows only by a factor of two. We are able to assign possible driving sources to half of the outflows. Brighter MHOs are more likely to have a source candidate assigned to them.

Foreground star counts to GMCs associated with the outflows and the Besancon Galaxy model are used to determine distances. The method is calibrated using objects from the RMS survey. This allows us to estimate distances with a scatter of $25 \%$ (Ioannidis \& Froebrich 2012). The spatial distribution of the outflows shows that they follow the GMC complexes in the GP. However, there are large areas with high extinction where no flows are detected. About $2 / 3$ of the survey area is more or less devoid of outflows. The flows typically occur in groups of $3-5$ members with a size of $5 \mathrm{pc}$. The scale height of flows is $\sim 25-30 \mathrm{pc}$, similar to young clusters and OB stars. The 1-0S $(1)$ luminosities range from a few $10^{-4} \mathrm{~L}_{\odot}$ to $0.1 \mathrm{~L}_{\odot}$, ten times brighter than in nearby SF regions. Our sample is complete for objects brighter than $10^{-3} \mathrm{~L}_{\odot}$ at $5 \mathrm{kpc}$. This corresponds to HH211 behind $A_{K}=1 \mathrm{mag}$. The luminosity distribution of the outflows is a power law with $N \propto L_{\mathrm{H}_{2}}^{-1.9}$ and the integrated luminosities indicate a Milky Way SF rate (averaged over a typical jet lifetime) of more than $0.4 \mathrm{M}_{\odot} \mathrm{yr}^{-1}$. The energy and momentum of the jets is insufficient to sustain the typical local turbulence levels in their parental clouds.

The projected jet length drops exponentially in number for longer jets. The fraction of parsec scale flows is $25 \%$, twice as high as in nearby SF regions. A Monte-Carlo type model of jet speeds of $40-130 \mathrm{kms}^{-1}$ and ages between $4-20 \times 10^{3} \mathrm{yrs}$ can reproduce the length distribution. These lifetimes are an order of magnitude below estimates for the protostellar phase. For typical jet speeds the time gaps between the ejection of larger amounts of material are about $10^{3} \mathrm{yrs}$. According to the burst mode of SF models the creation of $\mathrm{H}_{2}$ knots is hence linked to low level fluctuations of the mass accretion rate and not FU-Ori type events. Their duty cycle is more in agreement with the total jet lifetime, which suggests these outburst as trigger (or stopping point or both) of a jet ejection phase. However, better constraints of the FU-Ori duty cycle and mechanism as well as more detailed models are required to draw any further conclusions.
\end{abstract}

Keywords. ISM: jets and outflows — stars: formation

\section{References}

Froebrich, D., Davis, C. J., et al. 2011, MNRAS, 413, 480

Ioannidis, G. \& Froebrich, D. 2012, MNRAS, 421, 3257

Ioannidis, G. \& Froebrich, D. 2012, MNRAS, 425, 1380 